\title{
Mediación parental y habilidades digitales de los adolescentes de la Comunidad de Madrid: competencias y desempeño
}

\section{Parental mediation and digital skills of adolescents in the Community of Madrid: skills and performance}

María Cruz López-de-Ayala. Universidad Rey Juan Carlos. España.

mariacruz.lopezdeayala@urjc.es

$[\mathrm{CV}] \odot \mathrm{R}^{\mathrm{O}} \mathrm{O}$

Cristina Ponte. Universidade NOVA de Lisboa. Portugal.

cristina.ponte@fcsh.unl.pt

$[\underline{\mathrm{CV}}]$ (1) G R

Rebeca Martín-Nieto. Universidad Rey Juan Carlos. España.

rebeca.martin@urjc.es

$[\mathrm{CV}] \bigcirc \mathrm{R}^{\circ} \mathrm{O}$

Programa Nuevos Escenarios de Vulnerabilidad Digital: Alfabetización Mediática para una sociedad inclusiva PROVULDIG2-CM (H2019/HUM-5775) y el proyecto nacional "Redes sociales, adolescentes y jóvenes: convergencia de medios y cultura digital” (CSO2016-74980-C2-2-R)

La traducción del manuscrito en inglés se financió con fondos de la FCT - Foundation for Science and Technology (Portugal) en el marco del proyecto Ref: UIDB / 05021/2020.

Cómo citar este artículo / Referencia normalizada

López-de-Ayala, M.C., Ponte, C., Martín-Nieto, R. (2021). Mediación parental y habilidades digitales de los adolescentes de la Comunidad de Madrid: competencias y desempeño. Revista Latina de Comunicación Social, 79, 111-132. https://www.doi.org/10.4185/RLCS-2021-1523

\section{RESUMEN}

Introducción: Un uso positivo de internet y de los medios sociales, aprovechando sus oportunidades o afrontando sus peligros, requiere de habilidades digitales. La mediación parental es considerada un factor crítico para que los adolescentes adquieran estos conocimientos y habilidades digitales, adoptándolos en su comportamiento cotidiano. Metodología: A partir de los datos de una encuesta a una muestra representativa de 524 alumnos matriculados en Educación Secundaria Obligatoria (1216 años) de la Comunidad de Madrid (España) y aplicando análisis de regresión lineal jerárquica por pasos, este artículo examina las relaciones entre las diferentes formas de mediación parental reportadas por los adolescentes, por un lado, y sus competencias digitales y desempeño, por otro. Resultados: El análisis mostró que tanto la mediación parental restrictiva como la mediación parental orientada hacia el bienestar de los menores impactan, aunque débilmente, en las competencias online de los adolescentes: La primera limita el desarrollo de competencias digitales, mientras que la segunda las favorece. Sin embargo, la edad y el tiempo de uso influyen más en la puesta en práctica de competencias digitales. El co-uso guiado por los padres no se manifiesta como 
una práctica autónoma de la mediación para asegurar el bienestar, ni parece tener impacto sobre competencias y desempeño digital. Conclusiones: Los resultados sugieren que las prácticas parentales restrictivas y las prácticas familiares orientadas a advertir y aconsejar a los menores sobre los riesgos y buenos usos online no son más relevantes que los factores relacionados con la edad y la experiencia de uso en el desempeño digital.

PALABRAS CLAVE: Mediación parental; habilidades digitales; desempeño digital; conocimiento digital; adolescentes; Internet; redes sociales.

\begin{abstract}
Introduction: A positive use of the internet and social media, taking advantage of its opportunities and coping with its dangers, requires digital skills. Parental mediation is considered a critical factor for children and adolescents to acquire digital knowledge and skills, performing them in daily behavior. Methodology: Controlling variables such as sex, age, time of use and age of initiation in social networks, this article examines the relationship between different forms of parental mediation reported by Spanish adolescents, on the one hand, and their digital skills and performance, on the other. Using data from a survey of a representative sample of 524 pupils from1st to 4th year of Compulsory Secondary Education (from 12 to 16 years) in the Autonomous Region of Madrid (Spain). Results: we found that both restrictive mediation and active mediation oriented towards safety and well-being impacts on the online skills of adolescents, albeit weakly: restrictive mediation limits the development of digital skills, while forms of active mediation strengthens those skills. However, age and time of use influence more their implementation. Conclusions: The results suggest that, for adolescents' digital performance, either restrictive parental practices or parental practices warning about risks and advising good online uses are not more relevant than factors related to age and experience of use.
\end{abstract}

KEYWORDS: Parental mediation; digital skills; digital performance; digital knowledge; adolescents; Internet; social networks.

\title{
CONTENIDO
}

1. Introducción y estado de la cuestión. 2. Objetivos. 3. Metodología. 4. Resultados. 5. Discusión. 6. Conclusiones. 7. Referencias

\section{Introducción y estado de la cuestión}

\subsection{Habilidades digitales: usos y riesgos}

La relación de los adolescentes con Internet y las tecnologías digitales es un campo que ha atraído la atención de los investigadores, quienes se han interesado particularmente por los riesgos y posibles vulnerabilidades a los que los menores se pueden ver expuestos. Así mismo, se ha investigado qué factores condicionan esta exposición y cómo afrontar estas situaciones, adquiriendo protagonismo la mediación parental. En España, los datos sobre uso digital apoyan este interés: 94,5\% de los adolescentes de 12 a 15 años acceden habitualmente a Internet (INE, 2020); 71,5\% entre 12 y 13 años usa redes sociales, el 78\% de ellos con un perfil propio (AIMC, 2018); entre 14 y 16 años, el porcentaje con un perfil propio en redes sociales asciende al 92\% (Ballesteros y Picazo, 2018).

La extensión de los dispositivos móviles ha supuesto un salto cualitativo en este uso online, adelantando notablemente la edad de acceso (Garmendia et al., 2016), y derivándolo, posteriormente, hacia las redes sociales y la mensajería instantánea (Pastor-Ruiz et al., 2019). El consumo precoz y 
habitual de dispositivos conectados no implica que los usuarios sepan desenvolverse adecuadamente online (Livingstone et al., 2011), aunque se expongan a más riesgos conforme aumenta su exposición (Ólafsson et al., 2013) y hagan un uso más privado lejos de la supervisión de progenitores o docentes.

Para hacer un uso positivo de internet, evitando o afrontando adecuadamente sus peligros y aprovechando los beneficios que este ofrece, se requieren ciertas habilidades que faciliten la participación y el uso seguro en la red (Rodríguez-de-Dios e Igartua, 2016; Festl, 2020), cobrando relevancia la alfabetización mediática y digital. Leung y Lee (2012) sostienen que los adolescentes con mayor alfabetización digital perciben que están más preparados para evitar los riesgos de Internet. Jeong et al. (2012) confirman que las intervenciones de alfabetización mediática reducen las conductas de riesgo o antisociales y aumentan la autoeficacia para evitarlas. boyd y Hargittai (2010) han verificado su correlación con la configuración y gestión de la privacidad online. Otros estudios, sin embargo, no encuentran relación entre habilidades digitales y riesgos (Cabello-Hutt et al., 2017) o muestran una relación positiva entre ambos (Rodríguez-de-Dios et al., 2018).

Una revisión sistemática reciente de 110 estudios publicados en inglés en los últimos diez años, utilizando métodos cuantitativos y directamente relacionados con las habilidades digitales de los jóvenes de 12 a 17 años, presenta una fuerte evidencia de que las habilidades digitales de los niños mejoran con la edad, aunque se cuestiona si es debido al desarrollo de habilidades cognitivas y sociales o de la experiencia acumulada; si bien las diferencias de género son inconsistentes, existe una correlación positiva entre el rendimiento académico del niño y sus habilidades digitales; los niños con actitudes positivas hacia las TIC tienen mayores habilidades digitales (Haddon et al., 2020). Además de señalar la falta de consenso con respecto a la definición de habilidades digitales, el informe también subraya la necesidad de distinguir "las habilidades digitales demostradas o reivindicadas de la autoeficacia digital" (Haddon et al., 2020: 5): mientras que las primeras se revelan a través de prueba de desempeño o encuestas de autoinforme que hacen preguntas directas y fácticas, el segundo está sujeto a sesgos de deseabilidad.

Por otra parte, también conviene diferenciar entre competencias y desempeño digital, ya que se pueden adquirir los conocimientos y habilidades técnicas que permiten una mejor gestión de los recursos digitales, pero no hacerlo por diferentes motivos. Aunque la competencia y el desempeño digitales están manifiestamente vinculados, siendo la primera la base del segundo, Trultzsch-Wijnen (2020) subraya que la transferencia de una a otra no sucede directamente, sino que hay diversos factores individuales y ambientales que modelan esta relación.

Tomando en cuenta las competencias y los desempeños reportados por los adolescentes, este estudio se propone examinar la influencia de modalidades de mediación sobre las habilidades digitales.

\subsection{Mediación parental y habilidades digitales}

La mediación parental se puede entender como la gestión de los padres de la relación de sus hijos con los medios de comunicación (Livingstone y Helsper, 2008), incluyendo las interacciones paternofiliales (Sasson y Mesch, 2019) para controlar, supervisar e interpretar los contenidos de los medios.

En la literatura de la mediación parental online se partió de la clasificación utilizada con la televisión, que distingue tres estrategias: restrictiva, activa/instructiva/evaluativa y co-uso (Valkenburg et al., 1999). La primera se refiere a normas y prohibiciones respecto al tiempo y acceso a contenidos, la segunda se vincula con la discusión sobre los contenidos y la tercera supone el uso conjunto sin 
discusión. Sin embargo, en su aplicación a Internet, se valoró que los medios digitales no favorecen el uso compartido sin discusión (Livingstone y Helsper, 2008), y o bien se obvia el co-uso o bien se recoge incluido en la mediación activa como una estrategia única (Troseth, et al., 2016).

En este sentido, la mediación activa se entiende, principalmente, como el diálogo de los padres con sus hijos sobre los riesgos y usos digitales, ofreciéndoles consejos y orientación (Sonck et al., 2013). Sin embargo, cabe pensar que advertir a los menores de los riesgos online no siempre implica diálogo, y puede tener efectos muy diferentes al aprendizaje conjunto.

Mientras que algunos estudios exploran la efectividad de la mediación parental buscando una simplificación de estrategias (véase Symons et al, 2017), continúan apareciendo propuestas de mediación activa (véase Zaman et al., 2016; Glatz et al., 2018) que pueden resultar más efectivas. Cabe destacar el aprendizaje participativo, que supone la interacción y aprendizaje conjunto de padres e hijos a través de las tecnologías digitales (Clark, 2011), o las peticiones de apoyo parental iniciadas por los niños (Livingstone et al., 2017).

No obstante, no hay que perder de vista la influencia de la edad y el sexo de los menores sobre la actuación parental. Eastin et al. (2006) concluyeron que los padres dedican más tiempo y establecen más restricciones con los hijos varones y más jóvenes; Bartau et al. (2020) detectan una tendencia a combinar diferentes estrategias de mediación con los niños más mayores, sin ceñirse a una concreta, y una mayor mediación activa de los padres varones con sus hijas.

Aunque escasean los estudios longitudinales que examinan las variaciones en la mediación parental según la edad de los menores, los estudios existentes apuntan a que antes de la preadolescencia se aplican principalmente la supervisión y el co-uso (Eastin et al., 2006), orientándose progresivamente hacia la mediación activa y restrictiva (Nikken y Schols, 2015; Beyens et al., 2019); posteriormente, la intervención parental se relaja entre los adolescentes maduros (Sonck et al., 2013; Chen y Chng, 2016; Glatz et al., 2018; Ponte y Batista, 2020), cuando aumenta la resistencia a la supervisión paterna y las tareas de control se complican (Livingstone et al., 2017).

Otros estudios han explorado variables vinculadas con la edad de los menores, como la percepción paterna del nivel de uso que hacen sus hijos de Internet (Nikken y Schols, 2015), de sus habilidades y competencias online (Livingstone et al., 2017), el autocontrol (Li et al, 2019) o las reacciones de los niños (Haddon, 2015).

Estudios internacionales han mostrado como la mediación parental también está modelada por el contexto sociocultural. El reciente informe EU Kids Online muestra que los padres españoles se decantan más por sugerir formas para que sus hijos utilicen internet de forma segura $(65 \%)$ y ayudarles cuando algo les molesta (55\%) que por alentarles a explorar y aprender cosas con Internet (47\%), donde se sitúan muy por debajo del promedio del conjunto de países europeos (Smahel et al., 2020). En otras palabras, nos enfrentamos a una cultura de protección, especialmente con las niñas (Martínez et al., 2020), en lugar de una formación para las habilidades y el desempeño.

Se ha demostrado que la intervención paterna afecta a los riesgos y oportunidades online (Smahel et al., 2020). La mediación restrictiva reduce la actividad online de los menores, limitando los riesgos y las oportunidades online (Duerager y Livingstone, 2012). Esta estrategia reduce su eficacia con el avance de la adolescencia porque se puede entender como una intromisión en la privacidad y falta de confianza (Erickson et al., 2016). Los estudios empíricos muestran resultados menos consistentes de los efectos de la mediación activa sobre los riesgos (Soh et al., 2018; Bartau et al., 2018). 
Aunque se atribuye a los padres la responsabilidad de mantener a sus hijos seguros en su relación con los medios, se suele omitir que los padres también pueden guiar a sus hijos en su exploración en línea y mejorar su alfabetización digital (Sánchez-Valle et al., 2017). Esto es especialmente así si tenemos en cuenta que la incorporación de los padres al mundo digital y de los medios sociales conlleva que estos se estén convirtiendo en mediadores más capacitados digitalmente para apoyar y fomentar las habilidades digitales de sus hijos (López-de-Ayala et al., 2019). Sin embargo, pocos estudios han examinado el efecto de esta intervención sobre las habilidades digitales de los menores (Rodríguezde-Dios et al., 2018), y los resultados no son concluyentes.

Los escasos estudios que analizan cómo influye la mediación parental en las habilidades digitales de sus hijos (Rodríguez-de-Dios et al., 2018) concluyen que la mediación restrictiva reduce las habilidades de los menores (Sánchez-Valle et al., 2017; Glüer y Lohaus, 2018; Rodríguez-de-Dios et al., 2018). Erickson et al. (2016) añaden que las restricciones en las interacciones en línea en los adolescentes (13-17 años) pueden limitar la oportunidad de desarrollar habilidades para afrontar y resolver situaciones problemáticas. Sin embargo, los resultados con respecto a la mediación activa no son concluyentes. Recientemente, Livingstone et al. (2017) señalan que la estrategia de mediación habilitante es utilizada en mayor medida por los padres y niños más pequeños con más habilidades digitales; siendo esta una estrategia en la que tiene un fuerte peso la mediación activa de uso y de seguridad de internet, pero también con un componente importante de control técnico y supervisión. Cabello-Hutt et al. (2017) encontraron una asociación positiva, pero débil entre mediación activa y habilidades digitales en los niños de 9 a 17 años, y Rodríguez-de-Dios et al. (2018) no encontraron vinculación entre ambas en estudiantes de secundaria españoles (12-18 años). La revisión de la investigación internacional muestra que la mediación habilitante se asocia a mejores habilidades digitales, aunque algunos estudios no encuentran relación (Haddon et al., 2020: 6).

Se requieren, por tanto, más estudios que aclaren la relación entre habilidades digitales y las formas de mediación parental, teniendo en cuenta las nuevas propuestas de mediación activa y factores como la experiencia acumulada online o el desarrollo de responsabilidades en la adolescencia, como una fase de la vida que incluye la tarea de alcanzar la independencia emocional de los padres y de otros adultos (Havighurst, 1972). Más concretamente, nuestro objetivo es probar el carácter explicativo del uso conjunto de redes sociales guiado por los padres, como una práctica emergente (López de Ayala et al., 2019) y diferenciada de la mediación activa que se basa exclusivamente en el diálogo, para dotar a los menores de competencias que les permitan enfrentarse a diversas situaciones, algunas conflictivas, en estas plataformas.

\section{Objetivos}

De acuerdo con lo expuesto, este trabajo analiza la influencia de la mediación parental en la adquisición de habilidades digitales en los adolescentes, distinguiendo entre tres formas de mediación activa (asegurar el bienestar; co-uso orientado al aprendizaje guiado por los padres; y mediación solicitada por los hijos) y la mediación restrictiva. De este objetivo general, se derivan los siguientes objetivos:

1. Identificar las características demográficas y vinculadas al uso digital que influyen en los tipos de mediación.

2. Analizar cómo influyen los diferentes tipos de mediación activa y la mediación restrictiva en las competencias y el desempeño digital de los menores.

3. Identificar si el co-uso guiado por los padres constituye una práctica diferenciada de la mediación para garantizar el bienestar basada en el diálogo; examinando si la primera y la mediación iniciada por los hijos influyen sobre las competencias y el desempeño digital. 


\section{Metodología}

\subsection{Muestra}

Los datos analizados provienen de una encuesta a una muestra representativa de 524 alumnos matriculados en Educación Secundaria Obligatoria de la Comunidad de Madrid, una de las diecisiete comunidades autónomas que conforman el Estado Español y que alberga la capital de España. Su población estimada a 1 de enero de 2019 es 6.641 .648 de habitantes $(14,15 \%$ de población residente en España) (INE, 2020). Se siguió un muestreo polietápico estratificado (titularidad del centro público, privado o concertado-, y Madrid capital y el resto de los municipios) por conglomerados (centros educativos). En una primera etapa, se seleccionaron al azar 16 centros educativos y, en una segunda etapa, se seleccionaron las aulas. Los cuestionarios fueron administrados en los centros, utilizando el sistema de entrevista personal asistida por ordenador. Previamente, se había obtenido la autorización de los centros docentes y el consentimiento informado de padres ( $<14$ años) y el personal (igual o mayor a 14 años), tras informarles de las características de la investigación. El trabajo de campo fue realizado entre el 24 de octubre al 19 de diciembre de 2019. Después de eliminar a los mayores de 16 años (7 casos), la muestra final fue de $517\left(\mathrm{M}_{\text {edad }}=13,53, D T=1,19\right)$. El $48,6 \%$ eran chicas. La distribución por curso fue $27 \%$ de $1^{\circ}, 27 \%$ de $2^{\circ}, 25 \%$ de $3^{\circ}$ y $21 \%$ de $4^{\circ}$ de ESO. Finalmente, el $33 \%$ de los estudiantes provenían de centros públicos, el $44 \%$ de centros concertados y el $23 \%$ de centros privados.

El $12 \%$ de los adolescentes dijeron no utilizar las redes sociales, frente al $28 \%$ que lo hace continuamente, el $25 \%$ que lo hace todos los días y el $21 \%$ un rato todos los días; el resto lo hace 3-4 días a la semana (5\%), un día a la semana (3\%) o con menos frecuencia (5\%). Por último, el 50\% de los entrevistados se inició en las redes sociales a los 11/12 años; el 27\% lo hicieron con más de 12 años; el 16\%, a los 9/10 años; el 4,4, a los 7/8 años; y solo el 2\% lo hizo antes de los 7 años.

\subsection{Instrumentos de medida}

Las variables analizadas fueron: curso ( $1^{\circ}$ a $4^{\circ}$ de la ESO) (que es utilizado como un indicador de la edad), sexo ( 1 =hombre y $2=$ =mujer), tiempo de uso de redes sociales (No lo uso-continuamente; $1-7$ ), edad de inicio en redes sociales (Desde antes de los 7 años-más de 12 años, 1-5), habilidades digitales (competencias y desempeño), así como las estrategias de mediación parental.

Las habilidades online fueron analizadas con una escala propia que incorporaba una doble dimensión de conocimiento y aplicación, preguntando en qué medida conocían y realizaban acciones como "Bloquear el acceso de una persona que no me gusta en redes sociales", "Cambiar la configuración de privacidad en la red social", "Borrar el historial en el navegador", "Bloquear contenido no deseado", "Distinguir una página segura en la web", "Añadir una página a favoritos", "Navegar en incógnito o privado", "Poner o cambiar la contraseña de acceso al teléfono móvil/Smartphone", "Instalar aplicaciones" e "Informar de un abuso online". Las opciones de respuesta fueron: "Lo hago con frecuencia", "Lo he hecho alguna vez", "Sé hacerlo, pero nunca lo he hecho", "No sé cómo se hace" y "No sé lo que es/no lo entiendo".

Las respuestas fueron recodificadas en variables dummy, separando competencias digitales (Sé hacerlo=1; No sé hacerlo=0) y desempeño (No lo he hecho nunca=0; Lo he hecho alguna vez=1), y se sumaron los resultados para cada variable: competencias digitales (rango 1-10; $M=8,07 D T=2,55$; alfa de Cronbach=,857, $N=517$ ) y desempeño digital (rango $1-10 ; M=5,87 D T=2,57$; alfa de Cronbach=,758, $N=517$ ). 
Los ítems para la identificación de las estrategias de mediación parental se basan en una adaptación de las clasificaciones de Livingstone et al. (2011 y 2017) al análisis de las redes sociales, y en los resultados de un estudio cualitativo con entrevistas a padres de López-de-Ayala et al. (2019). La mediación parental para asegurar el bienestar se midió con los ítems: "Te avisan de los peligros de las redes sociales"; "Te comentan qué hacer si algo o alguien te molesta"; "Te dan consejos de cómo comportarte". El co-uso guiado por los padres con: "Veis o hacéis cosas juntos"; "Te enseñan a utilizar algunas aplicaciones y servicios y te dan consejos de cómo usarlos"; "Hablan contigo mientras aprendéis a hacer cosas juntos". Las escalas de respuestas de estos ítems oscilan entre 1 y 5 : desde "nunca" a "continuamente". La medición parental solicitada por los hijos incluía los ítems: "Cuando me ocurre algo que me molesta en redes sociales, se lo cuento a mis padres o les pido ayuda"; "A menudo consulto con mis padres las dudas sobre la información o contenidos que puedo subir a las redes sociales"; y "Pido ayuda a mis padres para utilizar aplicaciones o servicios". Las posibilidades de respuesta recogen cuatro opciones que abarcan desde "no se aplica a mi caso" a "se aplica totalmente a mi caso"). La mediación restrictiva se calculó sumando el número de prohibiciones parentales relativas al uso de las redes sociales reportadas por los entrevistados, y que incluyen: "Estar demasiado tiempo conectado"; "Hablar con desconocidos"; "Tener un perfil en cualquier red social"; "Tener un perfil en algunas redes sociales"; "Colgar fotos o videos personales"; y "Dar información personal". Las cuatro escalas alcanzaron niveles del Alfa de Cronbach que van desde 0,76 hasta 0,82 ; indicando una consistencia interna adecuada (estos resultados se pueden consultar en el epígrafe 3.1. y tabla 3).

\subsection{Análisis de datos}

Los datos han sido analizados con el programa estadístico SPSS (v.26), y el nivel de validez estadística se ha establecido para $p<, 05\left(p<, 01 * *\right.$ y $\left.p<, 05^{*}\right)$.

Se describió mediante porcentajes la utilización de las diferentes estrategias de mediación parental del uso digital de los adolescentes. A continuación, con el objetivo de confirmar la validez estructural de la escala de mediación parental relativa a los estilos de mediación activa, se realizó un análisis factorial exploratorio forzando tres dimensiones o factores, de acuerdo con la teoría (tabla 3). El objetivo era verificar la validez y fiabilidad de la escala en su aplicación a los estudiantes de la Comunidad de Madrid, identificando en qué medida el co-uso guiado por los padres se puede identificar como una estrategia independiente de mediación activa, en concreto, de la mediación parental para asegurar el bienestar.

La prueba estadística para estudiar las diferencias por sexo y curso, así como por tiempo de uso y edad de inicio en redes sociales, de las diferentes formas de mediación activa fue MANOVA, que permite la valoración de estas diferencias cuando las variables dependientes están relacionadas entre sí, limitando el error de tipo I, y que ha demostrado ser una prueba robusta ante el incumplimiento de los supuestos paramétricos (Bisquerra, 1989). Y esto debido a que las variables mediación para asegurar el bienestar, co-uso guiado por los padres y mediación solicitada por los hijos mantienen cierto grado de correlación entre ellas $\left(, 646^{* *}\right.$ para el primero y segundo, $503^{* *}$ para el segundo y tercero, y, $431 * *$ para el primero y tercero).

Los resultados de MANOVA, que indican si hay diferencias significativas por cada variable analizada cuando se toman conjuntamente las distintas modalidades de mediación, se reportan en el pie de las tablas 2 y 3 . Estos datos incluyen el valor la $\mathrm{F}$ (que nos indica el poder discriminante de esa variable) y su estadístico para contrastar la hipótesis nula de igualdad, sabiendo que cuanto mayor es el valor de la $\mathrm{F}$ y menor su significación, más probabilidad de que existan diferencias significativas entre los grupos que conforman las categorías de esa variable. Además, se incluye el 
lambda de Wilks ( $\lambda$ Wilks), que valora la significación estadística de las diferencias multivariantes entre los grupos, y el tamaño del efecto con eta parcial al cuadrado $\left(\eta^{2}\right.$ parcial).

Posteriormente, se aplicaron test univariados ANOVA, con pruebas post-hoc de Bonferroni para cada variable dependiente, que fueron evaluados a un nivel alfa de ,025. Los datos relativos a estos tests se informan en el apartado estadísticos en el interior de las tablas en referencia a cada variable.

Para estudiar las diferencias en la mediación restrictiva por sexo se ha utilizado el estadístico t de Student (t-test) o test de Welch, según sean o no las varianzas iguales, calculando la d de Cohen (d) para indicar el tamaño del efecto $(<0,20=$ muy pequeño; $0,20-0,50=$ pequeño; $0-50-0,80=$ medio; $>0,80=$ fuerte) (Cohen, 1988); con el curso, tiempo de uso y edad de inicio en redes sociales se ha utilizado el coeficiente de correlación de Spearman $\left(r_{\mathrm{s}}\right)$, que nos indica también la dirección y el tamaño del efecto.

Para conocer la influencia de la mediación parental sobre las competencias y el desempeño, se aplicaron análisis de regresión lineal jerárquica por pasos, y se incluyeron las variables predictoras relativas a las formas de mediación parental; así como dos bloques adicionales con variables sociodemográficas (curso y sexo), y sobre la experiencia de los adolescentes con redes sociales (tiempo de uso y edad de inicio).

El modelo de regresión por pasos realiza una continua reevaluación de los predictores incluidos en el modelo, eliminando aquellos regresores que quedan explicados por los restantes (porque carecen de contribución específica propia) y evita problemas de colinealidad con las variables excluidas.

\section{Resultados}

\subsection{Mediación parental: prevalencia y análisis factorial exploratorio}

Las prácticas más comunes reportadas por los adolescentes se corresponden con la mediación parental para asegurar el bienestar, con porcentajes superiores al $44 \%$ de los encuestados que indican que sus padres las realizan continuamente o bastante a menudo. Mientras que las prácticas correspondientes al co-uso guiado por los padres se sitúan todas por debajo de esta proporción, con alrededor de un $29 \%$ de adolescentes que reportan que sus padres les enseñan a utilizan aplicaciones y servicios y les dan consejos prácticos de cómo usarlos y o hablan con ellos mientras aprenden a hacer cosas juntos con esa frecuencia (tabla 1). 
RLCS, Revista Latina de Comunicación Social, 79, 111-132

[Investigación] DOI: 10.4185/RLCS-2021-1523| ISSN 1138-5820| Año 2021

Tabla 1. Adolescentes que reportan mediación parental para asegurar el bienestar y co-uso guiado por los padres (en porcentajes horizontales)

\begin{tabular}{|l|c|c|c|c|c|}
\hline & $\begin{array}{c}\text { Continua- } \\
\text { mente }\end{array}$ & $\begin{array}{c}\text { Bastante a } \\
\text { menudo }\end{array}$ & $\begin{array}{c}\text { Algunas } \\
\text { veces }\end{array}$ & Rara vez & Nunca \\
\hline Mediación parental para asegurar el bienestar & & & & & \\
\hline 1. Te avisan de los peligros de las redes sociales & 35,5 & 28,7 & 21,6 & 4,6 & 9,5 \\
\hline $\begin{array}{l}\text { 2. Te comentan qué hacer si algo o alguien te } \\
\text { molesta }\end{array}$ & 22,3 & 22,4 & 25,9 & 12,7 & 16,7 \\
\hline 3. Te dan consejos de cómo comportarte & 28,3 & 27,6 & 17,8 & 11,1 & 15,3 \\
\hline Co-uso guiado por los padres & & & & & \\
\hline 1. Veis o hacéis cosas juntos & 19 & 23,8 & 28,6 & 13,7 & 15 \\
\hline $\begin{array}{l}\text { 2. Te enseñan a utilizar algunas aplicaciones y } \\
\text { servicios y te dan consejos de cómo usarlos }\end{array}$ & 12,9 & 15,7 & 23,1 & 18,7 & 29,6 \\
\hline $\begin{array}{l}\text { 3. Hablan contigo mientras aprendéis a hacer cosas } \\
\text { juntos en internet }\end{array}$ & 10,7 & 18,4 & 24,9 & 20,7 & 25,3 \\
\hline
\end{tabular}

Fuente: Elaboración propia

En la mediación parental solicitada por los hijos (tabla 2), el 44\% de los adolescentes admiten que recurrir a sus padres cuando algo les incomoda en las redes sociales se puede aplicar totalmente o bastante su situación, y cerca de un tercio así lo manifiesta para las consultas sobre qué subir a redes sociales o solicitarles ayuda para utilizar aplicaciones o servicios.

Tabla 2. Mediación parental solicitada por los hijos informada por los adolescentes. En porcentajes horizontales

\begin{tabular}{|c|c|c|c|c|}
\hline & $\begin{array}{c}\text { Se aplica } \\
\text { totalmente a } \\
\text { mi caso }\end{array}$ & $\begin{array}{c}\text { Se aplica } \\
\text { bastante a mi } \\
\text { caso }\end{array}$ & $\begin{array}{l}\text { Se ajusta un } \\
\text { poco a mi } \\
\text { caso }\end{array}$ & $\begin{array}{l}\text { No se } \\
\text { aplica a mi } \\
\text { caso } \\
\end{array}$ \\
\hline \multicolumn{5}{|l|}{ Medición parental solicitada por los hijos } \\
\hline $\begin{array}{l}\text { 1. Cuando me ocurre algo que me molesta en redes } \\
\text { sociales, se lo cuento a mis padres o les pido consejo }\end{array}$ & 21,1 & 23,1 & 23,2 & 32,7 \\
\hline $\begin{array}{l}\text { 2. A menudo consulto con mis padres las dudas sobre la } \\
\text { información o contenidos que puedo subir a las redes } \\
\text { sociales }\end{array}$ & 15,7 & 19,6 & 23,4 & 41,3 \\
\hline $\begin{array}{l}\text { 3. Pido ayuda a mis padres para utilizar aplicaciones o } \\
\text { servicios }\end{array}$ & 14,7 & 17,7 & 29,7 & 37,9 \\
\hline
\end{tabular}

Fuente: Elaboración propia

Respecto a la mediación restrictiva, en torno a dos tercios señala que sus padres les prohíben hablar con desconocidos (65\%) o dar información personal (62\%). Algo menos habitual es que les prohíban estar demasiado tiempo conectados (58\%), seguido a gran distancia por colgar fotos o videos personales (38\%); y menos de la cuarta parte indica que les prohíben tener un perfil en algunas redes sociales $(24 \%)$ o en cualquier red social $(21 \%)$.

El análisis factorial exploratorio con tres dimensiones (tabla 3) dio como resultado los factores: mediación parental para asegurar el bienestar (F2=ítems 1, 2 y 3), co-uso guiado por los padres (F3=ítems 4, 5 y 6) y mediación solicitada por los hijos ( $\mathrm{F} 1=1$ tems 7, 8 y 9). El eigenvalue del co-uso guiado por los padres es menor de 1 , lo que indica que esta estrategia continúa asociada en cierta 
medida a la mediación para asegurar el bienestar, y que es registrada habitualmente en la literatura como mediación activa. No obstante, se mantuvo esta clasificación de tres factores con el objetivo de poner a prueba en qué medida el co-uso guiado por los padres podría estar favoreciendo el desarrollo de habilidades digitales en los menores.

Tabla 3. Análisis factorial exploratorio de modalidades de mediación activa, y media y desviación típica de cada ítem

\begin{tabular}{|c|c|c|c|c|c|}
\hline & Media & DT & $\begin{array}{c}\text { Mediación } \\
\text { p/ } \\
\text { asegurar } \\
\text { bienestar }\end{array}$ & $\begin{array}{l}\text { Mediación } \\
\text { solicitada } \\
\text { por los hijos }\end{array}$ & $\begin{array}{l}\text { Co-uso } \\
\text { guiado por } \\
\text { los padres }\end{array}$ \\
\hline 1. Te avisan de los peligros de ls redes sociales & 3,76 & 1,25 & ,780 & & \\
\hline 2. Te comentan qué hacer si algo o alguien te molesta & 3,21 & 1,37 & ,788 & & \\
\hline 3. Te dan consejos de cómo comportarte & 3,42 & 1,40 & ,779 & & \\
\hline 4. Veis o hacéis cosas juntos & 3,18 & 1,31 & & & ,775 \\
\hline $\begin{array}{l}\text { 5. Te enseñan a utilizar aplicaciones/servicios y te dan } \\
\text { consejos }\end{array}$ & 2,64 & 1,38 & & & 619 \\
\hline 6. Hablan contigo mientras aprendéis a hacer cosas juntos & 2,68 & 1,32 & & & ,789 \\
\hline $\begin{array}{l}\text { 7. Cuando algo me molesta, se lo cuento a mis padres o } \\
\text { les pido consejo }\end{array}$ & 2,33 & 1,14 & & ,796 & \\
\hline $\begin{array}{l}\text { 8. A menudo consulto con mis padres dudas sobre la } \\
\text { información/contenidos que puedo subir }\end{array}$ & 2,10 & 1,11 & & ,863 & \\
\hline $\begin{array}{l}\text { 9. Pido ayuda a mis padres para utilizar aplicaciones o } \\
\text { servicios }\end{array}$ & 2,09 & 1,07 & & ,790 & \\
\hline Eigenvalues & & & 4,358 & 1,322 & ,773 \\
\hline Varianza explicada & & & 24,94 & 24,82 & 21,94 \\
\hline Rango & & & $1-5$ & $1-4$ & $1-5$ \\
\hline Media & & & 3,47 & 2,17 & 2,68 \\
\hline$\overline{D T}$ & & & 1,14 & 0,95 & 3,28 \\
\hline Alfa de Cronbach & & & 804 & 819 & ,755 \\
\hline
\end{tabular}

Método de extracción: análisis de componentes principales. Método de rotación: Varimax con normalización Kaiser. La solución convergió en 5 iteraciones y explica el 71,7\% de la varianza explicada. Prueba de esfericidad de Barlett significativa $(1928,152$; gl=36, Sig. =,000) e indicador de la adecuación del tamaño de la muestra Kaiser-Meyer-Olkin adecuado (,873).

Fuente: Elaboración propia

\subsection{Factores personales y de la experiencia online asociados a las estrategias de mediación}

Los análisis de cada estrategia de mediación parental mostraron que más que el sexo, la edad importa (véase Tabla 4). Las chicas reportan mayor iniciativa que los chicos en la solicitud de mediación parental y experimentan mayor mediación restrictiva, pero el efecto es pequeño. Por otra parte, los estudiantes de cursos inferiores manifiestan solicitar más mediación parental y experimentar más mediación restrictiva: ambas disminuyen conforme avanza el curso, aunque el efecto es más fuerte en la mediación restrictiva. De hecho, los indicadores de mediación restrictiva en cuarto curso, con adolescentes mayores, son aproximadamente la mitad de los valores del primer curso. No se observan diferencias significativas en el resto de las formas de mediación activa en base a las variables analizadas. 
RLCS, Revista Latina de Comunicación Social, 79, 111-132

[Investigación] DOI: 10.4185/RLCS-2021-1523| ISSN 1138-5820| Año 2021

Tabla 4. Estrategias de mediación parental según sexo y curso

\begin{tabular}{|c|c|c|c|c|}
\hline & $\begin{array}{c}\text { Mediación para } \\
\text { asegurar el bienestar }\end{array}$ & $\begin{array}{l}\text { Co-uso guiado por los } \\
\text { padres }\end{array}$ & $\begin{array}{l}\text { Mediación solicitada por } \\
\text { los hijos }\end{array}$ & Mediación restrictiva \\
\hline \multicolumn{5}{|l|}{ Sexo } \\
\hline Hombre & $\mathrm{M}=3,36, \mathrm{DT}=1,17$ & $\mathrm{M}=2,77, \mathrm{DT}=1,09$ & $\mathrm{M}=2,03 * *, \mathrm{DT}=0,93$ & $\mathrm{M}=2,42 * *, \mathrm{DT}=1,89$ \\
\hline Mujer & $\mathrm{M}=3,58, \mathrm{DT}=1,09$ & $\mathrm{M}=2,90, \mathrm{DT}=1,09$ & $\mathrm{M}=2,32 * *, \mathrm{DT}=0,94$ & $\mathrm{M}=2,96 * *, \mathrm{DT}=1,94$ \\
\hline Estadístico & $\begin{array}{c}\text { ANOVA: } \\
\mathrm{F}(1,54)=4,123, p=, 043, \\
\left.\eta^{2} \text { parcial }=0.008\right)\end{array}$ & $\begin{array}{c}\text { ANOVA: } \mathrm{F}(1,54)=, 439, \\
p=, 508, \\
\left.\eta^{2} \text { parcial }=0.001\right)\end{array}$ & $\begin{array}{c}\text { ANOVA: } \mathrm{F}(1,54)=11,743, \\
p<, 01, \eta^{2} \text { parcial }=0.022\end{array}$ & $\begin{array}{c}\mathrm{t}(515)=-3,16, p<, 001, \\
\mathrm{~d}=-0,28\end{array}$ \\
\hline \multicolumn{5}{|l|}{ Curso } \\
\hline $1^{\circ}$ & $\mathrm{M}=3,48 \mathrm{DT}=1,14$ & $\mathrm{M}=2,89, \mathrm{DT}=1,11$ & $\mathrm{M}=2,38, \mathrm{DT}=0,99$ & $\mathrm{M}=3,69 * *, \mathrm{DT}=1,70$ \\
\hline $2^{\circ}$ & $\mathrm{M}=3,51 \mathrm{DT}=1,17$ & $\mathrm{M}=2,91, \mathrm{DT}=1,13$ & $\mathrm{M}=2,67 * *, \mathrm{DT}=1,00$ & $\mathrm{M}=2,73^{* *}, \mathrm{DT}=1,80$ \\
\hline $3^{\circ}$ & $\mathrm{M}=3,52, \mathrm{DT}=1,16$ & $\mathrm{M}=2,74, \mathrm{DT}=1,10$ & $\mathrm{M}=2,10^{* *}, \mathrm{DT}=0,82$ & $\mathrm{M}=2,22 * *, \mathrm{DT}=2,01$ \\
\hline $4^{\circ}$ & $\mathrm{M}=3,33, \mathrm{DT}=1,07$ & $\mathrm{M}=2,78, \mathrm{DT}=1,02$ & $\mathrm{M}=1,86^{* *}, \mathrm{DT}=0,88$ & $\mathrm{M}=1,86^{* *}, \mathrm{DT}=1,68$ \\
\hline Estadístico & $\begin{array}{c}\mathrm{F}(3,53)=, 188, p=, 905 \\
\eta^{2} \text { parcial }=0.001\end{array}$ & $\begin{array}{c}\mathrm{F}(3,53)=, 987, p=, 399, \\
\eta^{2} \text { parcial }=0.006\end{array}$ & $\begin{array}{c}\mathrm{F}(3,53)=6.348, p<, 001, \\
\eta^{2} \text { parcial }=0.035\end{array}$ & $\mathrm{r}_{\mathrm{s}}=-0.346, p<, 001$ \\
\hline
\end{tabular}

Notas. **diferencia significativa para $p<, 01 \mathrm{y} *$ para $p<, 05$. MANOVA (mediación para asegurar el bienestar, co-uso guiado por los padres y mediación solicitada por los hijos) para sexo, $\mathrm{F}(3,524)=5,183, p<, 01 ; \lambda$ Wilks $=0,971, \eta^{2}$ parcial $=0.029$; y curso: $\mathrm{F}(9,16)=3,122, p<, 01 ; \lambda$ Wilks $=0,948, \eta^{2}$ parcial $=0.018$

Fuente: Elaboración propia

Respecto a las diferencias en las formas de mediación aplicadas según la experiencia de los adolescentes en redes sociales (tabla 5), se observan diferencias significativas de mediación restrictiva según el tiempo de uso (los niños que usan con menos frecuencia reportan valores más elevados de mediación restrictiva). Sin embargo, esta variable no parece influir en el resto de las modalidades de mediación parental. 
RLCS, Revista Latina de Comunicación Social, 79, 111-132

[Investigación] DOI: 10.4185/RLCS-2021-1523| ISSN 1138-5820| Año 2021

Tabla 5. Estrategias de mediación parental según tiempo de uso y edad de inicio en redes sociales

\begin{tabular}{|c|c|c|c|c|}
\hline & $\begin{array}{c}\text { Mediación para asegurar } \\
\text { el bienestar }\end{array}$ & $\begin{array}{l}\text { Co-uso guiado por los } \\
\text { padres }\end{array}$ & $\begin{array}{c}\text { Mediación solicitada } \\
\text { por los hijos }\end{array}$ & Mediación restrictiva \\
\hline \multicolumn{5}{|c|}{ Tiempo de uso } \\
\hline $\begin{array}{l}\text { Continua- } \\
\text { mente }\end{array}$ & $\mathrm{M}=3,29, \mathrm{DT}=1,22$ & $\mathrm{M}=2,62, \mathrm{DT}=1,09$ & $\mathrm{M}=2,04, \mathrm{DT}=0,99$ & $\mathrm{M}=1,83^{* *}, \mathrm{DT}=1,66$ \\
\hline $\begin{array}{l}\text { Varias veces } \\
\text { al día }\end{array}$ & $\mathrm{M}=3,51, \mathrm{DT}=1,07$ & $\mathrm{M}=2,93, \mathrm{DT}=1,14$ & $\mathrm{M}=2,07, \mathrm{DT}=0,85$ & $\mathrm{M}=2,54 * * \mathrm{DT}=1,78$ \\
\hline $\begin{array}{lr}\text { Un } & \text { rato } \\
\text { todos } & \text { los } \\
\text { días } & \\
\end{array}$ & $\mathrm{M}=3,71, \mathrm{DT}=1,05$ & $\mathrm{M}=2,99, \mathrm{DT}=1,11$ & $\mathrm{M}=2,36, \mathrm{DT}=0,90$ & $\mathrm{M}=2,69 * *, \mathrm{DT}=1,83$ \\
\hline $\begin{array}{l}\text { 3-4 días a la } \\
\text { semana }\end{array}$ & $\mathrm{M}=3,82, \mathrm{DT}=0,91$ & $\mathrm{M}=3,04, \mathrm{DT}=0,93$ & $\mathrm{M}=2,26, \mathrm{DT}=0,84$ & $\mathrm{M}=3,25^{* *}, \mathrm{DT}=1,61$ \\
\hline $\begin{array}{l}\text { Un día a la } \\
\text { semana }\end{array}$ & $\mathrm{M}=3,38, \mathrm{DT}=0,73$ & $\mathrm{M}=2,81, \mathrm{DT}=0,61$ & $\mathrm{M}=2,48, \mathrm{DT}=1,18$ & $\mathrm{M}=3,10 * *, \mathrm{DT}=2,43$ \\
\hline $\begin{array}{l}\text { Con menos } \\
\text { frecuencia }\end{array}$ & $\mathrm{M}=3,30, \mathrm{DT}=1,08$ & $\mathrm{M}=2,92, \mathrm{DT}=1,00$ & $\mathrm{M}=2,01, \mathrm{DT}=0,92$ & $\mathrm{M}=3,76^{* *}, \mathrm{DT}=1,89$ \\
\hline No lo uso & $\mathrm{M}=3,31, \mathrm{DT}=1,33$ & $\mathrm{M}=2,76, \mathrm{DT}=1,12$ & $\mathrm{M}=2,31, \mathrm{DT}=1,03$ & $\mathrm{M}=4,11 * *, \mathrm{DT}=1,84$ \\
\hline Estadístico & $\begin{array}{c}\mathrm{F}(5,432)=, 820, p=, 536 \\
\eta^{2} \text { parcial }=0.009\end{array}$ & $\begin{array}{c}\mathrm{F}(5,432)=, 820 \\
p=, 536, \eta^{2} \\
\text { parcial }=0.009\end{array}$ & $\begin{array}{c}\mathrm{F}(5,432)=, 815 \\
p=, 539, \eta^{2} \\
\text { parcial }=0.009\end{array}$ & $\mathrm{r}_{\mathrm{s}}=-, 393, p<, 01$ \\
\hline \multicolumn{5}{|c|}{ Edad de inicio } \\
\hline Antes 7 años & $\mathrm{M}=2,46^{* *}, \mathrm{DT}=1,38$ & $\mathrm{M}=2,40, \mathrm{DT}=1,49$ & $\mathrm{M}=1,93, \mathrm{DT}=1,38$ & $\mathrm{M}=0,86, \mathrm{DT}=0,78$ \\
\hline $\begin{array}{lll}\text { De } 7 & \text { a } & 8 \\
\text { años } & & \\
\end{array}$ & $\mathrm{M}=3,17, \mathrm{DT}=1,07$ & $\mathrm{M}=2,23, \mathrm{DT}=1,32$ & $\mathrm{M}=2,21, \mathrm{DT}=0,89$ & $\mathrm{M}=2,17, \mathrm{DT}=1,34$ \\
\hline $\begin{array}{ll}\text { De } & 9-10 \\
\text { años } & \end{array}$ & $\mathrm{M}=3,43, \mathrm{DT}=1,07$ & $\mathrm{M}=2,81, \mathrm{DT}=1,14$ & $\mathrm{M}=2,05, \mathrm{DT}=0,94$ & $\mathrm{M}=2,44, \mathrm{DT}=1,87$ \\
\hline $\begin{array}{l}\text { De11-12 } \\
\text { años }\end{array}$ & $\mathrm{M}=3,56 * * \mathrm{DT}=1,12$ & $\mathrm{M}=2,89, \mathrm{DT}=1,04$ & $\mathrm{M}=2,22, \mathrm{DT}=0,94$ & $\mathrm{M}=2,50, \mathrm{DT}=1,87$ \\
\hline+ de 12 años & $\mathrm{M}=3,54 * * \mathrm{DT}=1,04$ & $\mathrm{M}=2,93, \mathrm{DT}=1,04$ & $\mathrm{M}=2,14, \mathrm{DT}=0,87$ & $\mathrm{M}=2,53, \mathrm{DT}=1,84$ \\
\hline Estadístico & $\begin{array}{c}\mathrm{F}(4,432)=3,792, p=, 005 \\
\eta^{2} \text { parcial }=0.034\end{array}$ & $\begin{array}{c}\mathrm{F}(4,432)=1,959 \\
p=, 100, \eta^{2} \\
\text { parcial }=0.018\end{array}$ & $\begin{array}{c}\mathrm{F}(4,432)=2,046 \\
p=, 087, \mathrm{\eta}^{2} \\
\text { parcial }=0.019\end{array}$ & $p=, 197$ \\
\hline
\end{tabular}

Notas. Diferencia significativa en el nivel $p<, 01^{* *}$ y para $p<, 05^{*}$. MANOVA mediación para asegurar el bienestar, couso guiado por los padres y mediación solicitada por los hijos) para tiempo de uso, $\mathrm{F}(15,1187)=1,122, p=, 331 ; \lambda$ $\mathrm{Wilks}=0,962, \mathrm{\eta}^{2}$ parcial $=0.013 ; \mathrm{y}$ edad de inicio del uso de redes sociales, $\mathrm{F}(12,1137)=2,459, p=, 004 ; \lambda \mathrm{Wilks}=0,935, \eta^{2}$ parcial $=0.022$

Fuente: Elaboración propia

Por otro lado, se observa que los que se iniciaron en redes sociales antes de 7 años muestran los valores más bajos de mediación para asegurar el bienestar. Sin embargo, no sucede lo mismo con el resto de las formas de mediación, incluyendo la mediación restrictiva, donde no se observan diferencias significativas.

En resumen, la mediación restrictiva es menor entre los chicos, entre los alumnos de cursos más avanzados y entre los que usan más tiempo las redes sociales. La mediación solicitada por los hijos también se ve afectada por el sexo y curso de los adolescentes, en el mismo sentido que la mediación restrictiva: es superior entre las mujeres y disminuye conforme avanza el curso. La mediación para asegurar el bienestar únicamente manifiesta diferencias significativas según la edad de inicio en redes sociales: los que se iniciaron más tarde en estas plataformas experimentan más esta forma de mediación que se concreta en la forma de consejos y advertencias. Por último, el co-uso guiado por los padres no muestra diferencias significativas para ninguna de variables independientes consideradas. 


\subsection{Habilidades digitales: competencias y desempeño}

El siguiente paso fue evaluar la influencia de los diferentes tipos de mediación en las competencias y el desempeño digital de los menores. Se realizaron dos análisis de regresión lineal jerárquica por pasos en redes sociales, con el objetivo de controlar intermediaciones o interacciones de otras variables que inciden en la mediación parental. Se incluyeron tres bloques en el análisis: uno con las formas de mediación (modelo 1); un segundo con las variables sociodemográficas (modelo 1); y un tercero con las variables referentes a la experiencia de los menores en redes sociales (modelo 3 ).

Los resultados del análisis de la regresión (tabla 6) para las competencias digitales muestran que las formas de mediación (modelo 1 y 2) apenas explican la varianza en las competencias digitales: un $3 \%\left(\mathrm{R}^{2}\right.$ ajustado=,033), lo que resulta insignificante. Cuando se incluye el bloque de variables sociodemográficas, la capacidad explicativa del modelo 3 asciende hasta el $9 \%$ ( $\mathrm{R}^{2}$ ajustado=,086). Las variables relativas a la experiencia de los adolescentes en redes sociales (tiempo de uso y edad de inicio) se excluyen del modelo porque no son significativas.

Tabla 6. Influencia de las estrategias parentales de mediación sobre las habilidades digitales

\begin{tabular}{|l|c|c|c|c|c|}
\hline & R cuadrado & R cuadrado ajustado & Cambio en el R & F (g.1.) & $\begin{array}{c}\text { Sig. cambio } \\
\text { en F }\end{array}$ \\
\hline \multicolumn{7}{|c|}{ Competencias digitales } \\
\hline Modelo 1 &, 022 &, 020 &, 022 & $9,804(1,439)$ &, 002 \\
\hline Modelo 2 &, 038 &, 033 &, 016 & $7,225(2,438)$ &, 007 \\
\hline Modelo 3 &, 092 &, 086 &, 052 & $26,008(3,437)$ & $<, 001$ \\
\hline \multicolumn{7}{|c|}{ Desempeño digital } \\
\hline Modelo 1 &, 028 &, 026 &, 028 & $12,556(1,439)$ & $<, 001$ \\
\hline Modelo 2 &, 093 &, 089 &, 065 & $22,492(2,492)$ & $<, 001$ \\
\hline Modelo 3 &, 120 &, 114 &, 027 & $19,912(3,437)$ & $<, 001$ \\
\hline
\end{tabular}

Nota: el coeficiente de terminación ajustado ( $\mathrm{R}^{2}$ ajustado) indica el porcentaje de la variable dependiente (competencias y desempeño) explicado por la regresión. El cambio en la $\mathrm{R}^{2}$ indica la ganancia en la varianza explicada al incluir un nuevo bloque de variables. La significación del cambio de la F nos indica si compensa incluir un nuevo bloque $(<, 05)$.

Fuente: Elaboración propia

Dentro del bloque de estrategias parentales, solo la mediación para asegurar el bienestar y la mediación restrictiva explican las competencias digitales de los menores (véase tabla 7). La mediación restrictiva mantiene una asociación negativa con las competencias digitales: conforme aumenta la mediación restrictiva disminuyen las competencias digitales de los menores. La mediación para asegurar el bienestar, por el contrario, favorece la adquisición de competencias digitales.

De las características sociodemográficas (modelo 3), únicamente el curso resulta significativo. Al incluir esta variable se retira parte de la carga explicativa de la mediación restrictiva, aunque continúa siendo significativa en términos estadísticos. Sin embargo, el efecto de la mediación para asegurar el bienestar, aunque bajo, se mantiene casi intacto. 
RLCS, Revista Latina de Comunicación Social, 79, 111-132

[Investigación] DOI: 10.4185/RLCS-2021-1523| ISSN 1138-5820| Año 2021

Tabla 7. Influencia de las estrategias parentales de mediación sobre las competencias digitales

\begin{tabular}{|c|c|c|c|}
\hline & \multicolumn{3}{|c|}{ Parámetros de la regresión } \\
\hline Variables & Beta estandarizado & $\mathrm{T}$ & Sig. \\
\hline \multicolumn{4}{|c|}{ Modelo 1} \\
\hline Mediación restrictiva &,- 148 & $-3,131$ & 002 \\
\hline \multicolumn{4}{|c|}{ Modelo 2} \\
\hline Mediación restrictiva &,- 187 & $-3,807$ &, 000 \\
\hline Mediación para asegurar el bienestar &, 132 & 2,688 & ,007 \\
\hline \multicolumn{4}{|c|}{ Modelo 3} \\
\hline Mediación restrictiva &,- 117 & $-2,357$ &, 019 \\
\hline Mediación para asegurar el bienestar & ,129 & 2,704 & 007 \\
\hline Curso & 243 & 5,100 &, 000 \\
\hline
\end{tabular}

Nota. Estadísticos de colinealidad adecuados: tolerancia y VIF con valores cercanos al 1, índice de condición por debajo de 10. Los coeficientes beta (número de unidades que aumentará la variable dependiente por cada unidad que aumente la variable independiente) se han estandarizado para determinar qué variables son más influyentes cuando se utilizan escalas diferentes.

\section{Fuente: Elaboración propia}

En conclusión, ninguna variable de experiencia digital influye sobre las competencias digitales. La influencia de la mediación para asegurar el bienestar y restrictiva resulta muy limitada (beta estandarizado de -,117 y ,129, respectivamente), mientras el curso (edad) manifiesta un poder predictivo moderado de las competencias digitales (beta estandarizado=,243).

Los resultados de la regresión que analizan los factores que prevén el desempeño digital son (tabla 4): el modelo 1, con las formas de mediación parental, apenas explica el 3\% de la varianza en el desempeño $\left(\mathrm{R}^{2}\right.$ ajustado=,026). En el modelo 2, que incluye el bloque de variables sociodemográficas de los adolescentes, la capacidad predictiva aumenta al $9 \%\left(\mathrm{R}^{2}\right.$ ajustado=,089). $\mathrm{Y}$ en el modelo 3, que incorpora el tiempo de uso y año de inicio en redes sociales, se alcanza el $11 \%$ de explicación $\left(\mathrm{R}^{2}\right.$ ajustado=,114).

En el modelo 1 (tabla 8), únicamente la mediación restrictiva muestra tener efectos: el desempeño disminuye cuando esta aumenta. En el modelo 2, el curso muestra una influencia sobre el desempeño digital: conforme se avanza de curso aumenta el desempeño. Además, anula la influencia de la mediación restrictiva, que ya no es significativa. Por último, en el modelo 3 , el tiempo de uso muestra efectos estadísticamente significativos, y el curso continúa manifestando una influencia moderada sobre las competencias digitales.

En definitiva, las variables que explican el desempeño digital por orden decreciente son: el curso (edad), con un efecto moderado sobre el desempeño (beta estandarizado=,259), y el tiempo de uso de redes sociales, con un efecto más débil (beta estandarizado=,172). Es decir, sería la edad y la experiencia medida en tiempo dedicado a redes sociales las únicas variables que predicen el desempeño digital, en tanto que las formas de mediación familiar no muestran efecto. 
RLCS, Revista Latina de Comunicación Social, 79, 111-132

[Investigación] DOI: 10.4185/RLCS-2021-1523| ISSN 1138-5820| Año 2021

Tabla 8. Influencia de las estrategias parentales de mediación sobre el desempeño

\begin{tabular}{|c|c|c|c|}
\hline \multirow[b]{2}{*}{ Variables } & \multicolumn{3}{|c|}{ Parámetros de la regresión } \\
\hline & Beta Estandarizado & $\mathrm{T}$ & Sig. \\
\hline \multicolumn{4}{|c|}{ Modelo 1} \\
\hline Mediación restrictiva &,- 167 & $-3,543$ &, 000 \\
\hline \multicolumn{4}{|c|}{ Modelo 2} \\
\hline Mediación restrictiva &,- 091 & $-1,917$ & 056 \\
\hline Curso & ,267 & 5,617 &, 000 \\
\hline \multicolumn{4}{|c|}{ Modelo 3} \\
\hline Mediación restrictiva &,- 046 &,- 945 & ,345 \\
\hline Curso &, 259 & 5,525 &, 000 \\
\hline Tiempo de uso de redes sociales &, 172 & 3,670 &, 000 \\
\hline
\end{tabular}

Nota: Los estadísticos de colinealidad son adecuados: con valores de tolerancia y VIF cercanos al 1.

Fuente: Elaboración propia

\section{Discusión}

Los resultados de las relaciones entre las diferentes formas de mediación parental reportadas por los adolescentes que cursan los cuatro años de educación secundaria obligatoria (12-16 años) y sus habilidades y desempeño digitales, incluidos los de seguridad y control de riesgos en las redes sociales, no pueden dejar de leerse teniendo en cuenta las particularidades de la relación con los padres en esta etapa de la vida, y las tareas de desarrollo (Havighurst, 1972) que se le asocian. Pero también hay que leerlos teniendo en cuenta el contexto más amplio del país y su cultura: en este sentido los resultados recogidos entre los estudiantes de la Comunidad de Madrid están en línea con los resultados de muestras representativas de España, que apuntan a una cultura de la protección parental, en lugar de un entrenamiento para habilidades y desempeño, tal y como apuntaba Martínez et al. (2020).

Entre las formas de mediación restrictiva, las tres primeras revelan preocupaciones consolidadas sobre los riesgos de la exposición en línea: la prohibición de hablar con extraños es reportada por casi dos de cada tres adolescentes, seguida de la prohibición de dar información personal. Ambas prohibiciones desafían la tarea del desarrollo en la adolescencia, que es lograr nuevas y más maduras relaciones con los pares de ambos sexos (Havighurst, 1972), que hoy pasa por las redes sociales y que requiere el manejo instrumental y crítico de las habilidades informacionales, sociales y expresivas, y el equilibrio entre el manejo de la privacidad y la participación (véase Helsper et al. 2021). Este pánico de los padres ilustra el mito del "stranger danger" e ignora el deseo de socialización y la cultura de los pares, donde la abrumadora mayoría de los nuevos contactos ocurren con personas de la misma edad y proporcionan gran satisfacción (Smahel et al., 2020). En tercer lugar, está la preocupación por el exceso de tiempo en línea $(58,2 \%)$, una preocupación de que el contexto que se produciría (debido a la pandemia) seguramente se reconfigurará. Además, alrededor de una quinta parte de los adolescentes denuncia la prohibición de los padres de tener un perfil en las redes sociales.

Si los resultados apuntan el impacto de la mediación restrictiva de los padres en las competencias digitales reportadas por adolescentes, este efecto resulta débil y desaparece sobre el desempeño cuando se controla el curso (la edad) y el tiempo de uso, que son los que marcan la diferencia. Esta mediación restrictiva está marcada por el sexo y la edad y parece extenderse desde la niñez, sugiriendo una cultura consistente de restricción del uso de las redes sociales, que se extiende hasta 
la adolescencia. Cuanto más se siente la mediación restrictiva, menos competencias digitales reportan los adolescentes, lo que concuerda con los resultados de Sánchez-Valle et al. (2017), Glüer y Lohaus (2018) y Rodríguez-de-Dios et al. (2018). Este resultado confirma que, si esta mediación puede proteger contra los riesgos digitales, al hacerlo tampoco les permite saber cómo lidiar con ellos y desarrollar las competencias adecuadas; tal que como sugieren Erickson et al. (2016) o Duerager y Livingstone (2012), entre otros. Que esto ocurra en un grupo de edad marcado por una fuerte cultura de pares y el deseo de autonomía ante la presión y el control familiar es un resultado que merece ser destacado.

Por otra parte, el co-uso guiado por los padres no se presenta como una estrategia autónoma de la mediación para asegurar el bienestar. Además, en contra de lo esperado y tras forzar su separación en el análisis, no se observa que esta impacte sobre las competencias o el desempeño digital.

Entre los tres tipos de mediación activa (iniciada por los padres y orientada al bienestar digital de los niños; co-uso guiado por los padres; iniciada por los niños), los adolescentes que empezaron a utilizar las redes sociales más temprano mencionan menos la primera, como si sus padres les hubieran dejado a ellos mismos explorar este nuevo espacio vital. La iniciativa de los padres se afirma en el reconocimiento de lo que es importante hacer. Los resultados relativos al efecto de la mediación orientada al bienestar sobre las habilidades autoinformadas son consistentes con los alcanzados por Cabello-Hutt et al. (2017) con niños de 9 a 17 años, que resultó en una asociación débil, frente a la ausencia de vinculación de los resultados del estudio de Rodríguez-de-Dios et al. (2018). Sin embargo, en nuestro estudio, ninguna de las fórmulas de mediación activa parece tener una influencia directa en el desempeño, es decir, en el acto de hacer.

En resumen, si las prácticas parentales restrictivas condicionan negativamente las competencias digitales, las prácticas familiares respaldadas por la iniciativa de un lado u otro no se muestran más relevantes que los factores relacionados con la edad. Nuevamente, el grupo de edad (los adolescentes) puede ser un factor de diferencia, no solo en relación con las competencias sino también con el desempeño.

Estos resultados son coherentes con los estudios que indican que las habilidades digitales varían según la edad (Haddon et al., 2020): únicamente el curso, que se puede equiparar con la edad, muestra una influencia tanto sobre las habilidades como en las competencias digitales.

En este trabajo partíamos de la premisa de que las formas de co-uso guiado por los padres podría marcar una diferencia positiva en el desarrollo de competencias digitales entre los adolescentes que les permitieran protegerse de los riesgos online respecto a la mediación parental para asegurar el bienestar, cuyos indicadores están claramente guiados por la orientación y el asesoramiento de los padres (te avisan, te comentan, te dan consejos...). Y la educación dialógica, que favorece la autonomía y la capacitación de los adolescentes, podría tener relación con la mayor involucración de los padres en las redes sociales que les facultan para implementar estas formas de mediación más comprometidas (López de Ayala, 2019). Se trataba, en definitiva, de poner a prueba formas alternativas de mediación parental sugeridas en la literatura, como las propuestas de aprendizaje compartido sugeridas por Clark (2011), o las actividades iniciadas por los niños solicitando el apoyo y la interacción con sus padres de Livingstone et al. (2017). En nuestros resultados, sin embargo, se muestra que se mantiene la asociación entre el uso conjunto iniciado por los padres con la mediación basada en el diálogo; con niveles más bajos de esta primera, según la declaración de los menores. En cuanto a las variables que predicen el co-uso iniciado por los padres, los análisis realizados no nos permiten señalar diferencias significativas en su aplicación, quizás debido a sus bajos niveles de implementación. 
Estos datos están en la línea de los encontrados en EU Kids Online para España, en la que se muestra una menor incidencia de este uso compartido (Smahel et al., 2020). Tampoco se ha encontrado una influencia de esta práctica sobre las competencias ni en el desempeño digital de facto de los menores.

El aprendizaje iniciado por los hijos muestra diferencias significativas por sexo y curso: son las mujeres adolescentes y los más jóvenes los que en mayor medida buscan en los padres una guía en el uso online. Al igual que ocurría con la mediación anterior, no muestra tener efecto en el desarrollo de competencias y desempeño digital.

\section{Conclusiones}

Este artículo buscó examinar las relaciones entre las diferentes formas de mediación parental reportadas por los adolescentes que cursan los cuatro años de educación secundaria obligatoria (1216 años) y sus habilidades y desempeño digitales, incluidos los de seguridad y control de riesgos en las redes sociales. Las preguntas fueron apoyadas por una extensa revisión de la literatura sobre habilidades digitales, por un lado, y las perspectivas sobre la mediación parental, por el otro. Siguiendo estas perspectivas, los instrumentos de medición buscaron formalizar los temas de manera fáctica, evitando las falacias de la autoeficacia. Los instrumentos de medición en relación con la mediación parental también buscaban asegurar la comparabilidad de los resultados con otros estudios $\mathrm{y}$ formalizar nuevas preguntas.

Cabe señalar que todos los resultados, sobre la mediación de los padres, la seguridad y las habilidades de búsqueda de información y las acciones que toman o no toman en estas situaciones (su desempeño) se basan en respuestas autoinformadas de los adolescentes. Una evaluación de las actuaciones digitales se lograría mejor a través de respuestas a situaciones específicas. Nuevos trabajos pueden profundizar en el tema combinando los cuestionarios con experimentación en los que se midan las habilidades de facto de los adolescentes, en línea con uno de los objetivos del proyecto de investigación europeo ySKILLS, que busca revisar y reconceptualizar el conocimiento sobre habilidades digitales (cf www.yskills.eu). Como señalan Helsper et al. (2021), los aspectos funcionales se han medido más comúnmente que los aspectos críticos de las habilidades digitales, mientras que las habilidades relacionadas con la comunicación y la interacción y con la creación y producción de contenido se han investigado menos. El mismo proyecto considera en estos procesos de adquisición y consolidación de habilidades la atención al factor tiempo y cómo evolucionan las habilidades de los adolescentes. Se apunta así la necesidad de continuar investigando la influencia de diversos factores sobre el desempeño digital, no solo para la consolidación del conocimiento científico sino también para el diseño de políticas públicas y lineamientos adecuados para diferentes públicos, incluidas las familias.

\section{Referencias}

Asociación para la Investigación de Medios de Comunicación (AIMC) (Noviembre, 7th 2018). "Más del $40 \%$ de los niños ve contenidos televisivos en los dispositivos móviles o el ordenador. Estudio AIMC Niñ@s 2018” (Nota de prensa). http://bit.ly/2Sm1Gpe

Ballesteros, J.C. y Picazo, L. (2018). Las TIC y su influencia en la socialización de adolescentes. FAD. https://bit.ly/3130IdC 
Bartau, I., Aierbe, A. y Oregui, E. (2018). Mediación parental del uso de Internet en el alumnado de Primaria: creencias, estrategias y dificultades. [Parental mediation of the nternet use of Primary students: Beliefs, strategies, and difficulties]. Comunicar, 54(26), 71-79. https://doi.org/10.3916/C54-2018-07

Bartau, I., Aierbe, A. y Oregui, E. (2020). Mediación parental del uso de Internet desde una perspectiva de género. [Parental mediation of Internet use from a gender perspective]. Revista Electrónica de Investigación Educativa, $22, \quad \mathrm{e} 02, \quad 1-14$. https://doi.org/10.24320/redie.2020.22.e02.2075

Beyens, I., Valkenburg, P.M. y Piorowski, J.T. (2019). Developmental trajectories of parental mediation across early and middle childhood. Human Communication Research, 45, 226-250. https://doi.org/10.1093/hcr/hqy016

Bisquerra, R. (1989). Introducción conceptual al análisis multivariable. Un enfoque informático con los paquetes SPSS-X, BMDP, LISREL Y SPAD. PPU.

boyd, d. y Hargittai, E. (2010). Facebook privacy setting. Who cares? First Monday, 15(8). https://doi.org/10.5210/fm.v15i8.3086

Cabello-Hutt, T., Cabello, P. y Claro, M. (2017). Online opportunities and risks for children and adolescents: The role of digital skills, age, gender, and parental mediation in Brazil. New Media \& Society, 20(7), 2411-2431. https://doi.org/10.1177/1461444817724168

Clark, L. S. (2011). Parental mediation theory for the digital age. Communication Theory, 21(3), 323-343. https://doi.org/10.1111/j.1468-2885.2011.01391.x

Cohen, J. (1988). Statistical power analysis for the behavioral sciences (2nd ed). L. Erlbaum Associates.

Duerager, A. y Livingstone, S. (2012). How can parents support children's internet safety? LSE. EU Kids Online. http://eprints.lse.ac.uk/42872

Eastin, M.S., Greenberg, B.S. y Hofschire, L. (2006). Parenting the internet. Journal of Communication, 56, 486-504. https://doi.org/10.1111/j.1460-2466.2006.00297.x

Erickson, L.B., Wisniewski, P., Xu, H., Carroll, J.M., Rosson, M.B. y Perkin, D.F. (2016). The boundaries between: Parental involvement in a teen's online world. Journal of the Association for Information Science and Technology, 67(6), 1384-1403. https://doi.org/10.1002/asi.23450

Festl, R. (2020). Social media literacy \& adolescent social online behavior in Germany. Journal of Children and Media, 15(2), 249-271. https://doi.org/10.1080/17482798.2020.1770110

Garmendia, M., Jiménez, E., Casado, M.Á. y Mascheroni, G. (2016). Riesgos y oportunidades en internet y uso de dispositivos móviles entre menores españoles (2010-2015). Red.es/Universidad del País Vasco. https://bit.ly/2GnJ31D

Chen, V.H.H. y Chng, G.S. (2016). Active and restrictive parental mediation over time: Effects on youths' self-regulatory competencies and impulsivity. Computers \& Education, 98, 206-212. https://doi.org/10.1016/j.compedu.2016.03.012 
Glatz, T., Crowe, E. y Buchanan, C.M. (2018). Internet-specific parental self-efficacy: Developmental differences and links to Internet-specific mediation. Computers in Human Behavior, 84, 8-17. https://doi.org/10.1016/j.chb.2018.02.014

Gluier, M. y Lohaus, A. (2018). Elterliche und kindliche Einschätzung von elterlichen Medienerziehungsstrategien und deren Zusammenhang mit der kindlichen Internetnutzungskompetenz [Parents' and children's perspectives of parental mediation strategies in association with children's internet skills]. Prax Kinderpsychol Kinderpsychiatr, 67(2),181-203. https://doi.org/10.13109/prkk.2018.67.2.181

Haddon, L. (2015). Children's critical evaluation of parental mediation. Cyberpsychology: Journal of Psychosocial Research on Cyberspace, 9(1), article 2. https://doi.org/10.5817/CP2015-1-2

Haddon, L., Cino, Davide, Doyle, M-A, Livingstone, S., Mascheroni, G. y Stoilova, M. (2020). Children's and young people's digital skills: a systematic evidence review. KU Leuven, Leuven: ySKILLS. https://zenodo.org/record/4274654\#.YGD2gS35TC8

Havighurst, RJ (1972). Developmental tasks and Education. Longman, New York \& London (Original: 1948)

Helsper, E. J., Schneider, L. S., van Deursen, A. J.A.M. y van Laar, E. (2021). The youth Digital Skills Indicator: Report on the conceptualisation and development of the ySKILLS digital skills measure. KU Leuven, Leuven: ySKILLS. http://doi.org/10.5281/zenodo.4608010

Instituto Nacional de Estadística (INE) (2020). Encuesta sobre equipamiento y uso de tecnologías de información y comunicación en los hogares. http://www.ine.es/

Jeong, S.H., Cho, H. y Hwang, Y. (2012). Media literacy interventions: A meta-analytic review. Journal of Communication, 62(3), 454-472. https://doi.org/10.1111/j.1460-2466.2012.01643.X

Li, J., Willems, Y.E., Stok, F.M., Dekivic, M., Bartels, M. y Kinkenauer, C. (2019). Parenting and Self-control across early to late adolescence: A Three-Level Meta-Analysis. Perspectives on Psychological Science, 14(6), 967-1005. https://doi.org/10.1177/1745691619863046

Leung, L. y Lee, P.S. (2012). The influences of information literacy, internet addiction and parenting styles on internet risks. New Media and Society, 14(1), 117-136. https://doi.org/10.1177/1461444811410406

Livingstone, S. y Helsper, E. (2008). Parental mediation and children's Internet use. Journal of Broadcasting \& Electronic Media, 52(4), 581-599. https://doi.org/10.1080/08838150802437396

Livingstone, S., Haddon, L., Görzig, A. y Ólafsson, K. (2011). Risks and safety on the internet. The perspective of European children. Full findings and policy implication form the EU Kids Online survey of 9-16 year olds and their parents in 25 countries. EU Kids Online. http://bit.ly/34igdIa

Livingstone, S., Olafsson, K., Helsper, E.J., Lupianez-Villanueva, F., Veltri, G.A. y Folkvord, F. (2017). Maximizing opportunities and minimizing risks for children Online: The role of digital skills in emerging strategies of parental mediation. Journal of Communication, 67, 82e105. https://doi.org/10.1111/jcom.12277 
López-de-Ayala, M.C., Martínez-Pastor, E. y Catalina-García, B. (2019). Nuevas estrategias de mediación parental en el uso de las redes sociales por adolescentes. El Profesional de la Información, 28(5). https://doi.org/10.3145/epi.2019.sep.23

Martínez, G., Casado, M. y Garitaonandia, C. (2020). Online parental mediation strategies in family contexts of Spain. [Estrategias online de mediación parental en contextos familiares de España]. Comunicar, 65, 67-76. https://doi.org/10.3916/C65-2020-06

Nikken, P. y Schols, M. (2015). How and why parents guide the media use of young children. Journal of Child and Family Studies, 24(11), 3423-3435. https://doi.org/10.1007/s10826-015$\underline{0144-4}$

Ólafsson, K., Livingstone, S. y Haddon, L. (2013). Children's use of online technologies in Europe. A review of the European evidence base. EU Kids Online. https://bit.ly/WH0wp8

Pastor-Ruiz, Y., Martín-Nieto, R. y Montes-Vozmediano, M. (2019). Patrones de uso, control parental y acceso a la información de los adolescentes en la red [Patterns of use, parental control and access to information for adolescents in the network]. Estudios sobre el Mensaje Periodístico, 25(2), 995-1012. https://dx.doi.org/10.5209/esmp.64821

Ponte, C. y Batista, S. (2020). Ambientes familiares e mediações digitais. In Ponte, C. (Coord..), Nós na rede. Ambientes digitais de crianças e jovens. Almedina \& ERC.

Rodríguez-de-Dios, I. y Igartua, J.J. (2016). Skills of digital literacy to address the risks of interactive communication. Journal of Information Technology Research, 9(1), 54e64. https://doi.org/10.4018/JITR.2016010104

Rodríguez-de-Dios, I., van Oosten, J. y Igartua, J.J. (2018). A study of the relationship between parental mediation and adolescents' digital skills, online risks and online opportunities. Computers in Human Behavior, 2, 186e198. https://doi.org/10.1016/j.chb.2018.01.012

Sánchez-Valle, M., de-Frutos-Torres, B. y Vázquez-Barrio, T. (2017). La influencia de los padres en la adquisición de habilidades críticas en Internet. [Parent's influence on acquiring critical Internet skills]. Comunicar, 53, 103-111. https://doi.org/10.3916/C53-2017-10

Sasson, H. y Mesch, G. (2019). Parental mediation. In R. Hobbs, \& P. Mihailidis (Eds.), The international encyclopedia of media literacy. Wiley Blackwell. https://doi.org/10.1002/9781118978238.iem10177

Smahel, D., Machackova, H., Mascheroni, G., Dedkova, L., Staksrud, E., Ólafsson, K., Livingstone, S. y Hasebrink, U. (2020). EU Kids Online 2020: Survey results from 19 countries. EU Kids Online. https://doi.org/10.21953/lse.47fdeqj01ofo

Soh, P.C., Chew, K.W., Koay, K.Y. y Ang, P.H. (2018). Parents vs peers' influence on teenagers' Internet addiction and risky online activities. Telematics and Informatic, 35, 225-236. https://doi.org/10.1016/j.tele.2017.11.003 
Sonck, N., Nikken, P. y de Haan, J. (2013). Determinants of internet mediation. A comparison of the reports by Duch parents and children. Journal of Children and Media, 7(1), 93-113. https://doi.org/10.1080/17482798.2012.739806

Symons, K., Ponnet, K., Emmery, K., Walrave, M. y Heirman, W. (2017). Parental knowledge of adolescents' online content and contact risks. Journal of Youth and Adolescence, 46(2), 401-416. https://doi.org/10.1007/S10964-016-0599-7

Troseth, G.L., Russo, C.E. y Strouse, G.A. (2016). What's next for research on young children's interactive media? Journal of Children and Media, 10(1), 54-62, https://doi.org/10.1080/17482798.2015.1123166

Trultzsch-Wijnen, C. (2020). Media Literacy. Discussing media socialization, agency and the appropriation of media. Springer

Valkenburg, P.M., Krcmar, M., Peeters, A.L. y Marseille, N.M. (1999). Developing a scale to assess three styles of television mediation: Instructive mediation, restrictive mediation, and social coviewing. Journal of Broadcasting \& Electronic Media, 4(1), 52-66. https://doi.org/10.1080/08838159909364474

Zaman, B., Nouwen, M., Vanattenhoven, J., de Ferrerre, E. y Van Looy, J. (2016). A qualitative inquiry into the contextualized parental mediation practices of young children's digital media use at home. Journal of Broadcasting and Electronic Media, 60(1), 11-22. https://doi.org/10.1080/08838151.2015.1127240

\section{AUTORES:}

\section{María Cruz López de Ayala López}

Doctora en Comunicación en la Universidad Rey Juan Carlos de Madrid (URJC) (2007), es Profesora Titular del área de la Facultad de Ciencias de la Comunicación en la URJC, y ha sido Visiting Fellow en London School of Economics and Political Sciences y en Universidade Nova de Lisboa. Sus principales líneas de investigación están relacionadas con: las audiencias jóvenes y adolescentes en Internet y redes sociales; menores, mediación parental y medios de comunicación. Ha participado en diferentes proyectos de investigación, tanto competitivos como art. 83, publicando en revistas científicas nacionales e internacionales.

mariacruz.lopezdeayala@urjc.es

Índice H: 12

Orcid ID: http://orcid.org/0000-0002-6989-866X

Google Scholar: https://scholar.google.es/citations?user=Hg59Qi4AAAAJ\&hl=es

ResearchGate: https://www.researchgate.net/profile/Maria-Lopez-118

Scopus ID: https://www.scopus.com/authid/detail.uri?authorId=55135803400

\section{Cristina Ponte}

Doctora en Ciencias de la Comunicación (2002), es Catedrática de Estudios de Media e Jornalismo en la Faculdade de Ciências Sociais e Humanas da Universidade NOVA de Lisboa (NOVA FCSH). Coordinadora del equipo portugués en la red EU Kids Online, contribuyó a la formación de la red Kids Online America Latina. Es actualmente miembro de coordinación del projecto europeo ySKILLS (2020-2014). Vicepresidenta del Grupo de Trabajo de ECREA sobre Children, Youth and Media (2012-2017) y de la Acción COST IS0906 (2010-2014). Coordinador de los proyectos 
Inclusión y Participación Digital (2009-2011), del Programa UT Austin-Portugal, y Children and Youth in the News (2005-2007), financiados por FCT. Autora/editora de 13 libros, tiene más de 40 artículos publicados en revistas internacionales y nacionales.

cristina.ponte@fcsh.unl.pt

\section{Índice H: 24}

Orcid ID: https://orcid.org/0000-0002-1534-4784

Google Scholar: https://scholar.google.es/citations?user=0X81PjQAAAAJ\&hl=es\&oi=ao

ResearchGate: https://www.researchgate.net/profile/Cristina-Ponte

Scopus ID: $\underline{\text { https://www.scopus.com/authid/detail.uri?authorId=35971390500 }}$

\section{Rebeca Martín Nieto}

Doctora en Ciencias de la Comunicación (2010) y Máster en Neurodidáctica. Profesora Contratada Doctor en la Facultad de Ciencias de la Comunicación (URJC). Sus principales líneas de investigación giran en torno a la comunicacion y la educación, así como al medio radiofónico. Ha participado en diferentes proyectos de investigación relacionados con la comunicación en las organizaciones y con la influencia de Internet en la infancia y la adolescencia. Forma parte del Grupo de Investigación Solidar\&dar y del de Investigación sobre Comunicación, Sociedad y Cultura (GICOMSOC).

rebeca.martin@urjc.es

\section{Índice $\mathrm{H}: 9$}

Orcid ID: https://orcid.org/0000-0003-3184-3564

Google Scholar: https://scholar.google.es/citations?user=8fJXAEAAAAAJ\&hl=es

ResearchGate: https://www.researchgate.net/profile/Rebeca-Nieto-2 